\title{
Event-related potentials of emotional memory: Encoding pleasant, unpleasant, and neutral pictures
}

\author{
FLORIN DOLCOS \\ Duke University, Durham, North Carolina \\ and University of Alberta, Edmonton, Alberta, Canada \\ and \\ ROBERTO CABEZA \\ Duke University, Durham, North Carolina
}

\begin{abstract}
Emotional events tend to be remembered better than nonemotional events. We investigated this phenomenon by measuring two event-related potential (ERP) effects: the emotion effect (more positive ERPs for pleasant or unpleasant stimuli than for neutral stimuli) and the subsequent memory effect (more positive ERPs for subsequently remembered items than for subsequently forgotten items). ERPs were measured while subjects rated the emotional content of pleasant, unpleasant, and neutral pictures. As was expected, subsequent recall was better for pleasant and unpleasant pictures than for neutral pictures. The emotion effect was sensitive to arousal in parietal electrodes and to both arousal and valence in frontocentral electrodes. The subsequent memory effect at centroparietal electrodes was greaterfor emotional pictures than for neutral pictures during an early epoch (400-600 msec). This result suggests that emotional information has privileged access to processing resources, possibly leading to better memory formation.
\end{abstract}

Emotional events tend to be remembered better than nonemotional events (Bradley, Greenwald, Petry, \& Lang, 1992; Christianson, 1992). The neural correlates of this memory-enhancing effect of emotion and their temporal aspects, in particular, are not well understood. Taking advantage of the temporal resolution of event-related potentials (ERPs), we investigated the time course of the electrophysiological correlates of encoding pleasant, unpleasant, and neutral pictures.

During the last decade, the field of cognitive neuroscience of emotion has grown dramatically. Most studies have focused on the perception and evaluation of emotional stimuli (emotional processing) and on the effects of emotion on memory formation (emotional memory). A critical distinction in this literature is the one between two affective dimensions: emotional arousal and emotional valence. Arousal refers to a continuum that varies from calm to excitement, whereas valence refers to a continuum that varies from pleasant to unpleasant, with neutral as an interme-

This research was supported by HFMR (Alberta, Canada) and NSERC (Canada); F.D. was supported by Chia PhD Scholarship and Dissertation Fellowship from the University of Alberta (Canada). We thank Annette Colangelo, Sanda Dolcos, and Eileen Noel for assistance with data collection and analysis and Kevin LaBar, Liz Phelps, and two anonymous reviewers for their comments on a previous version of the manuscript. Correspondence concerning this article should be addressed to F. Dolcos, Center for Cognitive Neuroscience, B203 LSRC, Duke University, Durham, NC 27708-0999 (e-mail: fdolcos@duke.edu). diate value (see Bradley \& Lang, 1994, for methods of assessing these two dimensions).

Different approaches ranging from behavioral and pharmacological to electrophysiological and functional neuroimaging have tried to define the anatomical and functional correlates of emotional processing and emotional memory (Cahill, 1996; Davidson \& Irwin, 1999; Lane \& Nadel, 2000; LeDoux, 1993; Phelps \& Anderson, 1997). In the following paragraphs, we briefly review studies in these two lines of research by presenting both general background information and specific ERP evidence. At the end of each section, we highlight the shortcomings of previous ERP studies and present the rationale for our approach.

\section{Neural Correlates of Emotional Processing}

Lesion (Adolphs, Tranel, Damasio, \& Damasio, 1994; Bechara, Damasio, Damasio, \& Lee, 1999), electrophysiological (Davidson \& Tomarken, 1989; Wheeler, Davidson, \& Tomarken, 1993), and functional neuroimaging (Canli, Desmond, Zhao, Glover, \& Gabrieli, 1998; Davidson, 1995; Davidson \& Irwin, 1999) evidence supports the role of the prefrontal cortex (PFC) and the amygdala in the evaluation of the emotional content of stimuli. The roles of other structures, such as ventral striatum, anterior cingulate, posterior parietal, and insula regions, have been also explored (Davidson \& Irwin, 1999; Lane \& Nadel, 2000). In the case of the PFC, different hypotheses have been proposed concerning the involvement of different $\mathrm{PFC}$ regions in emotional processes. The right-hemisphere 
hypothesis proposes that the right hemisphere is specialized for the perception, expression, and experience of emotion (e.g., Borod, Koff, \& Caron, 1983; see also Borod et al., 1998). The valence hypothesis proposes that the left hemisphere is primarily associated with processing of pleasant emotions, whereas the right hemisphere is primarily associated with processing of unpleasant emotions. This hypothesis is supported by electrophysiological (Davidson \& Tomarken, 1989) and functional neuroimaging (Canli et al., 1998; see also Davidson, 1995; Davidson \& Irwin, 1999) evidence. The amygdala has been strongly associated with emotional processing by both lesion (Adolphs et al., 1994) and functional neuroimaging (Irwin et al., 1996; Lane et al., 1997; Morris et al., 1996; Schneider et al., 1997) studies. For instance, lesion studies show that patients with bilateral amygdalar damage are impaired in detecting emotional facial expression (Adolphs et al., 1994), and imaging studies on neurologically intact people have reported amygdalar activations associated with processing of both pleasant and unpleasant stimuli (Irwin et al., 1996; Lane et al., 1997; Morris et al., 1996; Schneider et al., 1997).

ERP studies of emotion have identified an emotion ef$f e c t$ : ERPs for emotional stimuli (pleasant or unpleasant) tend to be more positive-going than ERPs for neutral stimuli (Carretie, Iglesias, \& Garcia, 1997; Cuthbert, Schupp, Bradley, Birbaumer, \& Lang, 2000; Johnston, Miller, \& Burleson, 1986; Naumann, Maier, Diedrich, Becker, \& Bartussek, 1997; Vanderploeg, Brown, \& Marsh, 1987). The emotion effect has been found in different ERP components, including the P300 component (e.g., Johnston et al., 1986; Naumann, Bartussek, Diedrich, \& Laufer, 1992; see also Diedrich, Naumann, Maier, \& Becker, 1997), the N300 component (Carretie, Iglesias, \& Garcia, 1997; Carretie, Iglesias, Garcia, \& Ballesteros, 1997), and the slow wave (SW) component (Cuthbert et al., 2000; Diedrich et al., 1997), but most consistently, the emotion effect is expressed in a P300-SW complex. The P300 component may be more sensitive to emotion under intentional emotional processing (e.g., Johnston et al., 1986; Naumann et al., 1992), whereas the N300 component appears to be more sensitive during incidental emotional processing (Carretie, Iglesias, \& Garcia, 1997; Carretie, Iglesias, et al., 1997). However, the evidence is inconclusive (Naumann et al., 1992). The neural generators of the emotion effect are not known, but it is reasonable to assume that it reflects interactions between the amygdala and cortical regions (Amaral, Price, Pitkanen, \& Carmichael, 1992; LaBar et al., 1999; Lane et al., 1997).

There are two main unsolved issues concerning the emotion ERP effect. First, it is unclear whether the emotion effect is sensitive only to arousal (emotional vs. neutral) or to both arousal and valence (pleasant vs. unpleasant). Whereas most studies have only found differences owing to arousal (see Diedrich et al., 1997, for a brief review), a few recent studies have also reported differences that could be attributed to emotional valence (Cuthbert et al., 2000; Diedrich et al., 1997). A possible explanation of why previous studies have not found clear differences owing to valence could be the use of word stimuli, which tend to generate smaller emotion effect overall $\left(3-4{ }_{\mu} \mathrm{V}\right.$ in amplitude; e.g., Naumann et al., 1997), as compared with more complex visual stimuli, such as pictures (5$6 \mu \mathrm{V}$ or more; e.g., Cuthbert et al., 2000). The failure to clearly dissociate the arousal and the valence could also be due to the fact that most studies either have compared only unpleasant and neutral items or have not equated the pleasant and the unpleasant stimuli for arousal. Also, many studies have used a relatively small number of stimuli per condition (20 or fewer), which may not give enough statistical power for fine dissociations. Second, if the emotion effect is sensitive to both arousal and valence, it is unclear whether these two dimensions can be dissociated in their topographical and/or temporal characteristics. There is some evidence that valence-related differences tend to be larger in anterior electrodes (Cuthbert et al., 2000; Diedrich et al., 1997), but most studies have employed too few electrodes (e.g., nine) to allow a clear dissociation in topography.

To explore these issues, we investigated the effect of arousal and valence on ERPs recorded from 32 electrodes while subjects rated the emotional content of 60 pleasant, 60 unpleasant, and 60 neutral pictures. Pictures were selected so that both pleasant and unpleasant pictures differed from neutral pictures in terms of both arousal and valence, whereas pleasant and unpleasant pictures differed from each other only in terms of valence.

\section{The Enhancing Effect of Emotion on Memory Formation}

Behavioral (Bradley et al., 1992), pharmacological (Cahill \& McGaugh, 1998; McGaugh, Cahill, \& Roozendaal, 1996), lesion (Adolphs, Tranel, \& Denburg, 2000; Cahill, Babinsky, Markowitsch, \& McGaugh, 1995; LaBar \& Phelps, 1998), and neuroimaging (Cahill et al., 1996; Canli, Zhao, Brewer, Gabrieli, \& Cahill, 2000; Canli, Zhao, Desmond, Glover, \& Gabrieli, 1999; Hamann, Ely, Grafton, \& Kilts, 1999) studies have suggested that the beneficial effect of emotion on memory is related to both arousal and valence, with arousal as the most critical factor. Pharmacological studies with animals have investigated the role of peripheral adrenergic systems and their interaction with other neurotransmitter systems (e.g., cholinergic, GABA-ergic, opiod-peptidergic)in the amygdala (Cahill \& McGaugh, 1998; McGaugh et al., 1996). The results of these studies have suggested that the amygdala exerts its enhancing role on memory for emotionally arousing stimuli through the modulation of hippocampal activity (Cahill \& McGaugh, 1998; McGaugh et al., 1996).

Lesion (Adolphs et al., 2000; Cahill et al., 1995; LaBar $\&$ Phelps, 1998) and functional neuroimaging (Cahill et al., 1996; Canli et al., 2000; Canli et al., 1999; Hamann et al., 1999) studies on humans have supported the role of the amygdala in the formation of emotional memory. For instance, lesion studies have shown that patients with 
bilateral amygdala damage do not remember emotional story segments better than the neutral ones (Cahill et al., 1995), and temporal-lobectomy patients, unlike control subjects, do not exhibit an increase in memory for arousing words over time (LaBar \& Phelps, 1998). Also, subjects with left-amygdalar damage are impaired in their memory for emotional stimuli, despite entirely normal memory for neutral stimuli (Adolphs et al., 2000). Imaging studies using emotional stimuli have shown that the number of emotional items remembered was correlated with activity in the amygdalar (Cahill et al., 1996; Hamann et al., 1999) and hippocampal (Hamann et al., 1999) regions. Also, a more recent event-related fMRI study (Canli et al., 2000) showed that left-amygdalar activity during encoding predicted memory for unpleasant, but not for neutral, pictures.

In the ERP literature, there are a considerable number of studies on emotion (for reviews, see Diedrich et al., 1997; Halgren \& Marinkovic, 1995) and on memory (for reviews, see Rugg, 1995; Wagner, Koutstaal, \& Schacter, 1999), but very few of them have investigated the modulatory effect of emotion on memory. Some of the latter have examined the effect of emotion on memory retrieval (e.g., Maratos, Allan, \& Rugg, 2000; Maratos \& Rugg, 2001; Windmann \& Kutas, 2001), but only a couple of studies focused on emotional encoding. One study (Palomba, Angrilli, \& Mini, 1997) measured ERPs during the encoding of emotional and neutral pictures and found that the amplitude of ERPs recorded from a parietal electrode (i.e., PZ) was positively correlated with the number of subsequently remembered slides. Another study (LaBar et al., 1999) combined ERP and blood flow (fMRI) measurements during the encoding of unpleasant and neutral pictures and identified a neural network (including the prefrontal, parietal, and occipitotemporal cortices) potentially involved in the encoding of unpleasant pictures.

A limitation of these studies is that they did not use what is probably the most powerful method available to investigate the neural correlates of memory encoding: the subsequent memory paradigm. In this paradigm, encoding trials are sorted according to whether the item presented in each trial was remembered or forgotten in a subsequent memory test (Brewer, Zhao, Desmond, Glover, \& Gabrieli, 1998; Paller, Kutas, \& Mayes, 1987; Wagner et al., 1998). ERPs for items that are subsequently remembered tend to be more positive-going than ERPs for items that are subsequently forgotten (e.g., Fabiani, Karis, \& Donchin, 1986; Paller et al., 1987; Sanquist, Rohrbaugh, Syndulko, \& Lindsley, 1980; for reviews, see also Rugg, 1995, and Wagner et al., 1999). This difference is known as the $s u b$ sequent memory effect (or Dm effect; Paller et al., 1987). It can occur over frontal, midline, or parietal locations, and it can onset as early as 300-400 msec and can persist beyond 1,200 msec (Rugg, 1995).

The main goal of the present study was to investigate the relationship between the emotion effect and the subsequent memory effect. When the memory advantage for emotional stimuli and the ERP positivity associated with both the emotion effect and the subsequent memory effect are considered together, it is reasonable to predict that the beneficial effect of emotion on memory performance will appear as an interaction between the emotion effect and the subsequent memory effect. That is, if emotional stimuli are better remembered because they are better encoded (as has been suggested by the aforementioned lesion, pharmacological, and neuroimaging evidence), the subsequent memory effect should be modulated by the emotional content of the encoded stimuli. Yet the only ERP study, to our knowledge, that directly investigated the subsequent memory effect for emotional stimuli (Leiphart, Rosenfeld, \& Gabrieli, 1993) failed to find a subsequent memory effect or a modulatory influence of emotion on the subsequent memory effect. This null finding could be related to two aspects of this study. First, as was suggested by the authors, it could be related to the use of a shallow encoding task, which contrasts with the use of deep encoding tasks in other subsequent memory studies (e.g., Paller et al., 1987). Second, the null finding could be related to the use of words as stimuli. As was mentioned above, words tend to elicit a weaker emotional effect than pictorial stimuli do, thereby reducing the chances of finding a significant emotion-related difference in the subsequent memory effect.

Thus, we investigated the modulatory influence of emotion on the subsequent memory effect, using a deep encoding task (pleasantness rating) and pictures as stimuli. ERPs were recorded while subjects rated random series of pleasant, unpleasant, and neutral pictures on a 5-point pleasantness scale. After the encoding phase, the subjects performed a free recall task in which they wrote a description for each picture they could remember. Encoding ERPs were separately averaged for pleasant, unpleasant, and neutral pictures and, within each category, for subsequently remembered and forgotten items.

We made two predictions concerning emotional processing, and two predictions concerning emotional memory. Concerning emotional processing, we expected that the emotion effect would be reflected mainly in the modulation of the P300-SW complex and would be sensitive to both arousal and valence and that valence-related differences would have an anterior distribution, possibly leading to clear topographical dissociation from arousalrelated differences (Cuthbert et al., 2000; Diedrich et al., 1997). Concerning emotional memory, we predicted that memory for emotional pictures would be better than that for neutral pictures (Bradley et al., 1992) and that, consistent with this behavioral difference, the subsequent memory effect would be more pronounced for emotional than for neutral pictures.

\section{METHOD}

\section{Subjects}

Fifteen healthy right-handed female university students participated in the experiment in exchange for payment or credits. Female subjects were chosen because previous studies using the same kind of stimuli have found that women show more physiological reac- 
tivity with valence judgments than do men (Lang, Greenwald, Bradley, \& Hamm, 1993) and because women are more likely to report intense emotional experiences (Shields, 1991). The experimental data were collected with the understanding and written consent of each subject, and the procedures used for data collection adhered to generally accepted practices for experimental research on human participants.

\section{Materials}

Stimuli consisted of a pool of 180 pictures (60 pleasant, 60 unpleasant, and 60 neutral) selected from the International Affective Picture System (IAPS; Lang, Bradley, \& Cuthbert, 1997). IAPS pictures are rated on a 9-point scale in terms of both arousal $(1=$ calm and $9=$ excited $)$ and valence $(1=$ unpleasant, $5=$ neutral, $9=$ pleasant), using the Self-Assessment Manikin method (Bradley \& Lang, 1994). The average arousal and valence scores of the selected pictures ${ }^{1}$ were, respectively, 6.0 and 7.1 for pleasant pictures, 6.2 and 2.3 for unpleasant pictures, and 2.9 and 5.0 for neutral pictures. Pleasant and unpleasant pictures differed from each other in terms of emotional valence, but not in terms of emotional arousal, whereas both pleasant and unpleasant pictures differed from neutral pictures in terms of both arousal and valence. These two facts were confirmed by two analyses of variance (ANOVAs). First, an ANOVA on the valence scores of pleasant, unpleasant, and neutral pictures yielded a significant main effect of valence $[F(2,118)=1,292.3$, $p<.0001]$, and post hoc tests showed significant differences between the valence scores of all three categories $(p<.05)$. Second, an ANOVA on the arousal scores of pleasant, unpleasant, and neutral pictures yielded a signif icant main effect of arousal $[F(2,118)=$ $1,246.2, p<.0001]$, and post hoc tests showed significant differences between the arousal scores of both the pleasant and the unpleasant pictures and those of the neutral pictures $(p<.05)$, but no significant difference between the pleasant and the unpleasant pictures $(p>.05)$. The pictures were formatted so that, on the screen, they had a height of between 10 and $13 \mathrm{~cm}$ and a width of between 10 and $17 \mathrm{~cm}$. The 180 pictures were divided into six sets of 30 pictures $(10$ pleasant, 10 unpleasant, and 10 neutral), which were assigned to six study-test blocks.

\section{Procedure}

The experiment was conducted on a PC using the SuperLab software (Cedrus Inc.). The experimental session consisted of a practice session and six study-test blocks. Eight different block orders were employed, and the order of the pictures within each block was randomized for each subject. Encoding trials consisted of four events: blank $(500 \mathrm{msec})$, cross fixation $(500 \mathrm{msec})$, picture $(2,000 \mathrm{msec})$, and rating screen (until response). The subjects were instructed to experience the feelings or thoughts elicited by each picture and to rate the pictures according to their initial reaction, using a 5-point scale $(1=$ very unpleasant, $2=$ slightly unpleasant, $3=$ neutral, $4=$ slightly pleasant, and $5=$ very pleasant ). The subjects were also instructed to remember the pictures for a subsequent memory test (intentional learning). After each study phase, the subjects were asked to recall as many pictures as possible during $6 \mathrm{~min}$. They were provided with response sheets and were instructed to write down a description for each picture, using one line per picture. They were asked to provide enough details so that an outsider could identify each picture (e.g., diver vs. skier) and could differentiate it from similar pictures (e.g., platform diver vs. springboard diver). Only pictures whose descriptions were detailed enough to allow both identification and differentiation were classified as remembered.

\section{ERP Recording}

EEG was recorded from $32 \mathrm{Ag} / \mathrm{AgCl}$ electrodes embedded in a cap (Quikcap, Neurosoft Inc., Sterling, VA), with respect to a linked mastoid reference. Recording sites were based on a variation of the 10/20 international electrode placement system (Jasper, 1958), in- cluding the following electrodes: frontopolar (FP1, FP2), frontal (F3, F7, FZ, F4, F8), frontocentral (FC3, FCZ, FC4), central (C3, CZ, $\mathrm{C} 4)$, centroparietal (CP3, CPZ, CP4), parietal (P3, PZ, P4), frontotemporal (FT7, FT8), temporal (T7, T8), temporoparietal (TP7, TP8), and occipital (O1, OZ, O2). Horizontal eye movements were monitored with bipolar electrodes placed symmetrically on the outer canthus of each eye, and vertical movements were monitored with bipolar electrodes situated on the left supraorbital and infraorbital ridges. All channels were amplified with a filter bandwith of $0.03-$ $50 \mathrm{~Hz}$ and were sampled on line at an A/D rate of $500 \mathrm{~Hz}$. The recording epoch was $1,500 \mathrm{msec}$, beginning $100 \mathrm{msec}$ prior to picture onset. Trials with values above $100 \mu \mathrm{V}$ or below $-100 \mu \mathrm{V}$, possibly owing to eye movement artifacts, were eliminated from the analysis. To ensure an adequate signal-to-noise ratio in the ERPs, subjects who had fewer than 16 artifact-free trials per condition were excluded from analysis and were replaced (a total of 4 subjects were excluded and replaced).

\section{RESULTS}

\section{Behavioral Results}

Valence ratings. The average valence scores of the pictures, as rated by the subjects, were 4.11 for pleasant pictures $(S D=0.37), 1.47$ for unpleasant pictures $(S D=$ $0.24)$, and 2.95 for neutral pictures $(S D=0.24 ; 1=$ very $u n$ pleasant, $3=$ neutral, and $5=$ very pleasant . An ANOVA on the valence rating scores of pleasant, unpleasant, and neutral pictures yielded a significant main effect of valence $[F(2,28)=312.48, p<.0001]$, and post hoc tests showed significant differences between the valence scores of all three categories (all $p$ s $<.01$ ). Thus, our subjects' rating scores were consistent with the standard valence scores from IAPS (see the Method section).

Memory performance. As was expected, memory performance was affected by the emotional content of the pictures. The proportion of pictures recalled was .57 for pleasant pictures $(S D=.091), .58$ for unpleasant pictures $(S D=.095)$, and .48 for neutral pictures $(S D=.078)$. A one-way (emotion: pleasant, unpleasant, and neutral) ANOVA yielded a significant main effect of emotion $[F(2,32)=16.44, p<.0001]$, and post hoc contrasts yielded significant differences between pleasant and neutral pictures $(p<.05)$ and between unpleasant and neutral pictures $(p<.05)$, but not between pleasant and unpleasant pictures $(p>.05)$. Thus, memory performance was higher for emotional than for neutral pictures, with no difference between pleasant and unpleasant pictures.

\section{ERP Results}

To investigate the emotion effect, ERPs were separately averaged for pleasant, unpleasant, and neutral pictures. To investigate the modulatory influence of emotion on the subsequent memory effect, ERPs in each emotional category were further subdivided into ERPs for remembered and forgotten items. Averaged data were analyzed using repeated measures ANOVAs and post hoc Tukey-Kramer tests.

Emotion effect. The overall shape of encoding ERPs was similar for pleasant, unpleasant, and neutral pictures, and it was characterized by a N200-N300-P300b- 


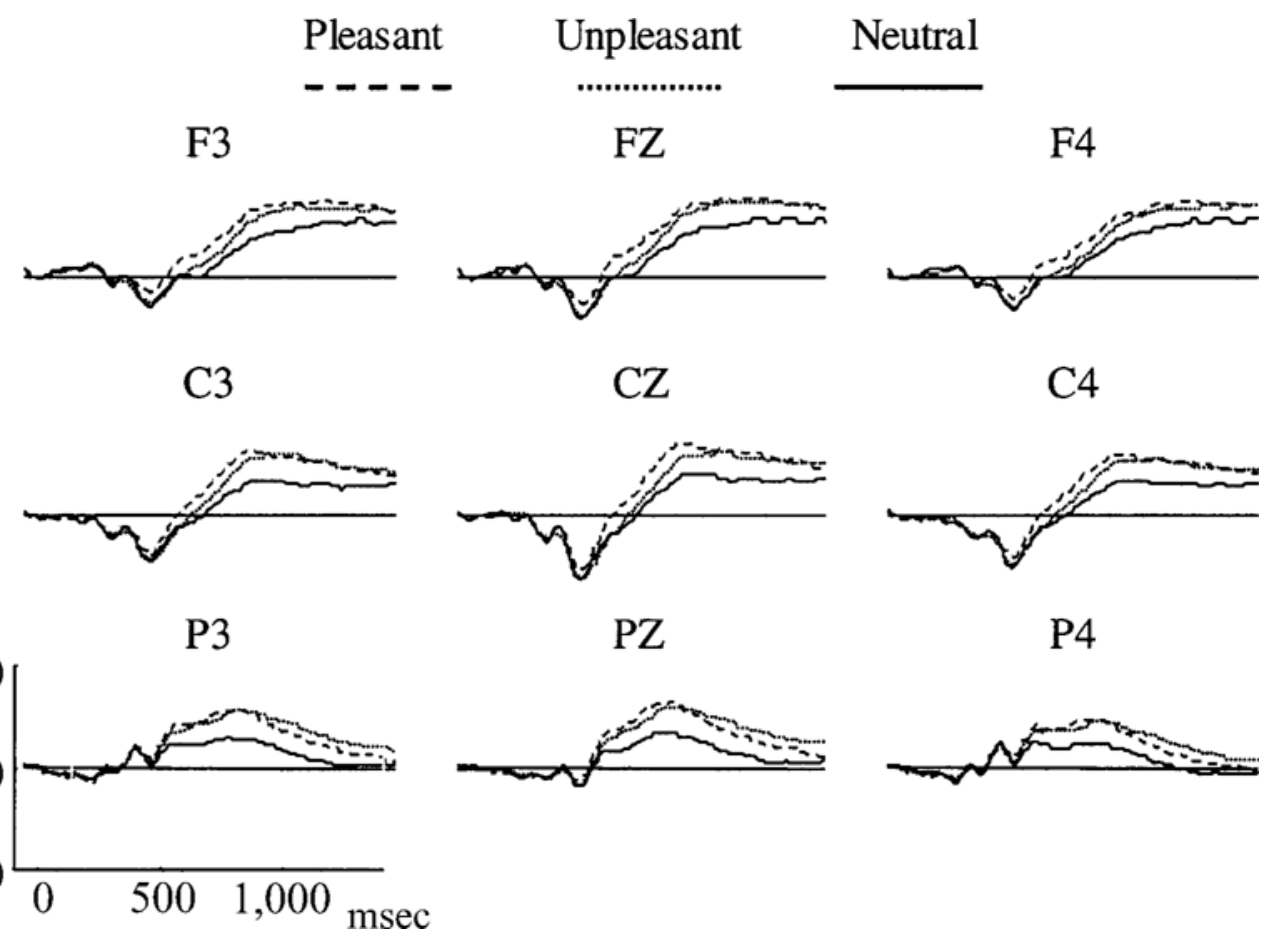

Figure 1. Emotion effect. ERPs for both pleasant and unpleasant pictures were more positive-going than ERPs for neutral pictures. The electrode locations are topographically arranged as viewed from above the subjects' heads. Vertical and horizontal scales represent the voltage amplitude (in microvolts) and the time from stimulus onset (in milliseconds), respectively.

SW complex (Figure 1). As was expected, there was an emotion effect: ERPs for emotional pictures were more positive-going than ERPs for neutral pictures. Overall, the emotion effect reflected mainly differences in arousal, which where most prominent in the amplitude of SWs and at parietal electrodes. However, during an earlier epoch (i.e., 500-800 msec) and at frontocentral electrodes (e.g., FCZ in Figure 2), the emotion effect was sensitive mainly to valence. As Figure 2 suggests, the emotion effect was different at parietal versus frontocentral electrodes. At parietal electrodes, the emotion effect appeared at the same time $(500 \mathrm{msec})$ for both categories of high-arousing pictures (i.e., pleasant and unpleasant), whereas at frontocentral areas, it appeared earlier for pleasant pictures $(500 \mathrm{msec})$ than for unpleasant pictures (after $800 \mathrm{msec}$ ). No noticeable valence-related hemispheric asymmetry was present (Figure 1).

To test these ideas, ANOVAs were computed on ERPs from PZ and FCZ, at two time windows (see Figure 2). These locations and time windows were chosen on the basis of previous evidence that showed a tendency for a posterior/anterior topographical dissociation of the arousal/valence effects, at similar locations and ERP components (Cuthbert et al., 2000).

To verify whether the emotion effect was different at parietal versus frontocentral electrodes at $500-800 \mathrm{msec}$, a 2 (electrode site: PZ vs. FCZ) $\times 3$ (emotion: pleasant, unpleasant, neutral) ANOVA was computed. It yielded significant main effects of both electrode site $[F(1,14)=$ $16.25, p<.0012]$ and emotion $[F(2,28)=27.84, p<$ $.0001]$ and a significant electrode site $\times$ emotion interaction $[F(2,28)=4.57, p<.019]$. To elucidate this interaction, separate one-way (pleasant/unpleasant/neutral) ANOVAs were computed on PZ and FCZ. At PZ, the ANOVA yielded a significant effect of emotion $[F(2,28)=$ $31.02, p<.0001$ ] , and the post hoc tests showed a significant difference between pleasant pictures and neutral pictures $(p<.05)$ and between unpleasant and neutral pictures $(p<.05)$, but not between pleasant and unpleasant pictures $(p>.05)$. At FCZ, in contrast, the ANOVA yielded a significant effect of emotion $[F(2,28)=18.29$, $p<.0001]$, and post hoc tests showed a significant difference between pleasant pictures and both unpleasant and neutral pictures $(p \mathrm{~s}<.05)$, but not between unpleasant and neutral pictures $(p>.05)$. Thus, the two separate ANOVAs confirmed a difference between emotional (both pleasant and unpleasant) and neutral pictures in parietal regions, but a difference between pleasant and both unpleasant and neutral pictures in frontocentral regions (compare FCZ and PZ graphs for the early epoch in Figure 2).

To verify whether the emotion effect at FCZ was different over time, a 2 (epoch: $500-800$ vs. $800-1,200 \mathrm{msec}) \times$ 3 (emotion: pleasant, unpleasant, and neutral) ANOVA was performed. It yielded significant main effects of both epoch $[F(1,14)=56.53, p<.0001]$ and emotion 
A. FCZ

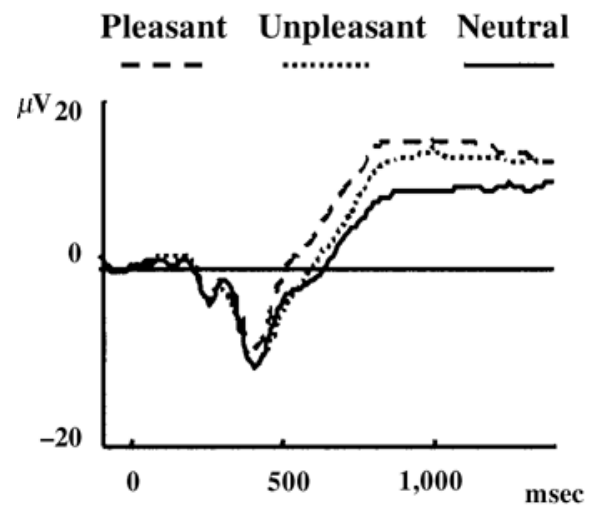

Early epoch $(500-800 \mathrm{msec}) \quad$ Late epoch $(800-1,200 \mathrm{msec})$
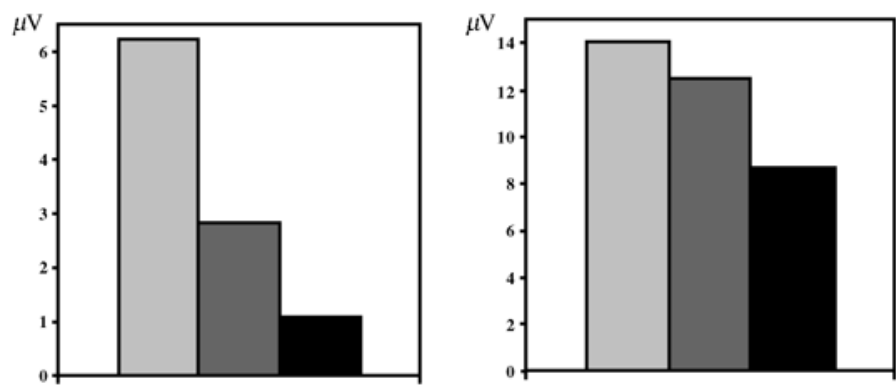

\section{B. PZ}

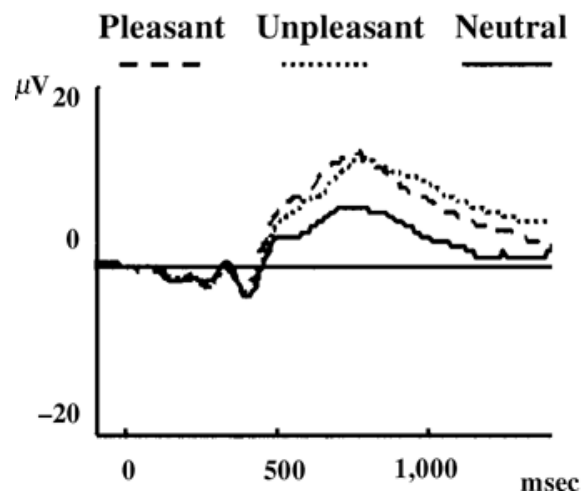

Early epoch $(500-800 \mathrm{msec}) \quad$ Late epoch $(800-1,200 \mathrm{msec})$
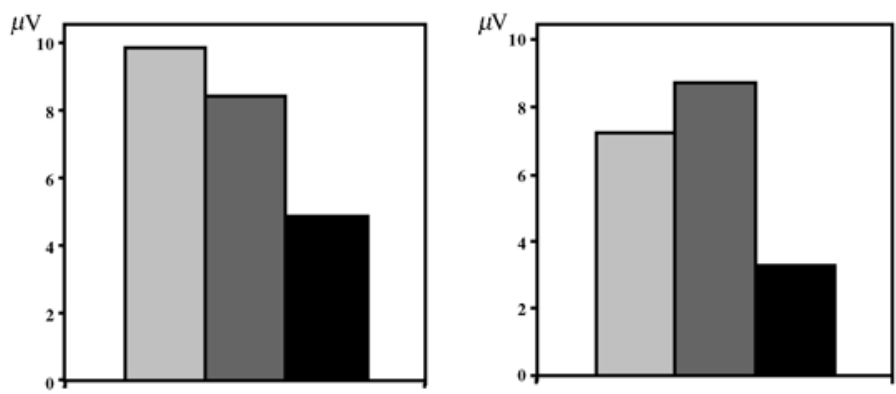

Figure 2. The emotion effect was different at frontocentral electrodes than at parietal electrodes. (A) At frontocentral electrodes, it appeared earlier for pleasant pictures (500-800 msec) than for unpleasant pictures (after $800 \mathrm{msec}$ ). (B) At parietal electrodes, it appeared at the same time $(\mathbf{5 0 0 - 8 0 0} \mathrm{msec})$ for both pleasant and unpleasant pictures. Light gray, pleasant; dark gray, unpleasant; black, neutral.

$[F(2,28)=22.6, p<.0001]$ and a significant epoch $\times$ emotion interaction $[F(2,28)=10.28, p<.0004]$. To further quantify these effects, an additional one-way (emotion: pleasant, unpleasant, and neutral) ANOVA was performed at the late epoch of FCZ. It yielded a significant main effect of emotion $[F(2,28)=25.24, p<.0001]$, and post hoc tests showed a significant difference for both pleasant and unpleasant pictures, when compared with neutral pictures $(p<.05)$, but no significant difference between pleasant and unpleasant pictures $(p>.05)$.

Finally, to check for hemispheric differences in the emotion effect, 3 (emotion: pleasant, unpleasant, and neutral) $\times 2$ (hemisphere: left vs. right) ANOVAs were computed on ERPs from left (F3, FP1) and right (F4, FP2) frontal locations. The ANOVAs performed on ERPs from F3/F4 did not yield a significant emotion $\times$ hemisphere interaction, neither during an early epoch (500$800 \mathrm{msec}$ ) nor during a late epoch $(800-1,200 \mathrm{msec})$, suggesting that the emotion effect at these locations was similar across hemispheres. However, the same $3 \times 2$ design computed on ERPs from FP1/FP2 and during the early epoch (500-800 msec) yielded a significant emo- tion $\times$ hemisphere interaction $[F(2,28)=3.94, p<.032]$, suggesting that the emotion effect was different across hemispheres during this early interval. Follow-up ANOVAs and post hoc analyses showed that this interaction was due to a larger emotion effect for pleasant pictures in the left hemisphere, as compared with the right $[F(1,14)=$ $11.23, p<.005]$. Although this finding is consistent with the valence hypothesis of hemispheric asymmetry (Davidson, 1995), because the analyses did not yield analogous results for unpleasant pictures (larger emotion effect in the right hemisphere, as compared with the left), it does not provide clear ERP evidence for the valence hypothesis. However, the fact that this lateralization was seen in the frontal electrodes is consistent with the proposed role of the frontal lobes in the processing of emotional valence (Davidson, 1995; Heller, 1993).

Taken together, these results show that the emotion effect was different at parietal versus frontocentral locations and suggest that, at parietal locations, the emotion effect was sensitive mainly to arousal (pleasant $=$ unpleasant $>$ neutral), whereas at frontocentral locations, it was sensitive mainly to valence during an earlier epoch (pleasant $>$ 


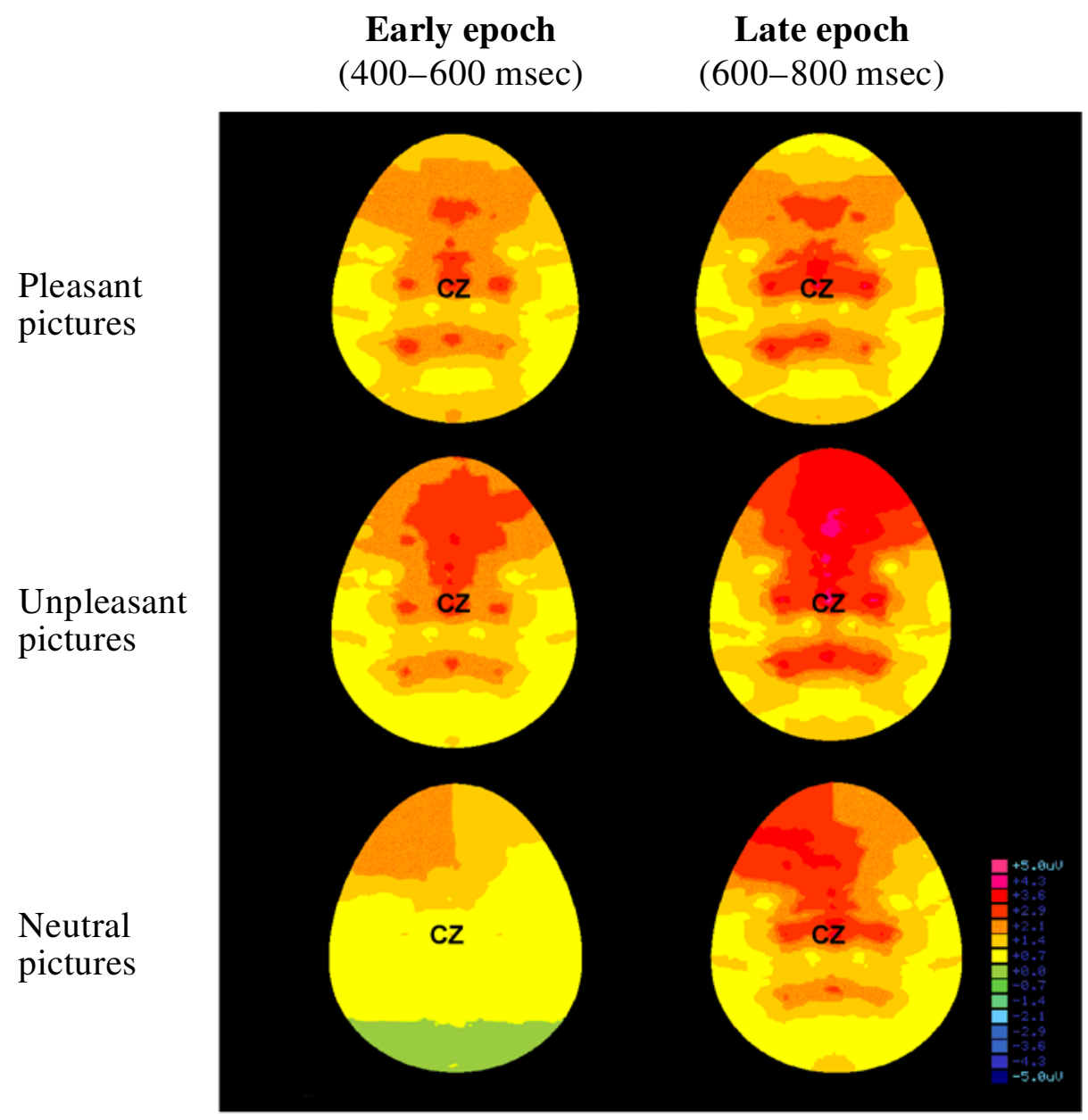

Figure 3. Subtraction (recalled - forgotten) topomaps comparing the subsequent memory effect for pleasant, unpleasant, and neutral pictures at different epochs. The subsequent memory effect over centroparietal areas occurred earlier for pleasant and unpleasant pictures $(400-600 \mathrm{msec})$ than for neutral pictures (after $600 \mathrm{msec}$ ). The maximal difference occurred at $\mathrm{CZ}$.

unpleasant $=$ neutral $)$ and then, later, to overall arousal (pleasant $=$ unpleasant $>$ neutral $)$.

Subsequent memory effect. As is illustrated by Figure 3 , the distribution of the subsequent memory effect was different during an early epoch than during a late epoch. During an early epoch (400-600 msec), the subsequent memory effect at midline locations (e.g., CPZ, $\mathrm{CZ}, \mathrm{FCZ}$ ) was greater for pleasant and unpleasant pictures than for neutral pictures. The difference was maximal at $\mathrm{CZ}$, and it can be clearly observed in the subtraction data (remembered - forgotten) depicted in Figure 4. During a late epoch (600-800 msec), however, the distribution of the subsequent memory effect was more similar for pleasant, unpleasant, and neutral pictures: It extended over centroparietal and frontocentral regions, and it was maximal at midline electrodes. In addition to the overall similarity in the general pattern of activation, some differences in the subsequent memory effect can also be noticed. As Figure 3 suggests, the subsequent memory effect at frontal electrodes seems to be bilateral for pleasant pictures, right-lateralized for unpleasant pictures, and left-lateralized for neutral pictures.

To investigate the effect of arousal on the latency of the subsequent memory effect, ANOVAs were conducted on subtraction data (remembered - forgotten) from CZ at two different epochs: 400-600 and 600-800 msec (see Figure 4). These locations and time windows were selected because, as was mentioned above, the subsequent memory effect is likely to occur at midline electrodes and as early as 300-400 msec after stimulus onset (Rugg, 1995). Since a preliminary ANOVA yielded no difference between pleasant and unpleasant pictures either at the early $[F(1,14)=0.002, p<.97]$ or at the late $[600-800 \mathrm{msec}$; $F(1,14)=0.11, p<.75]$ epoch, statistical power was increased by collapsing pleasant and unpleasant conditions into one emotional category. The 2 (epoch: 400-600 vs. 600-800 msec) $\times 2$ (arousal: emotional vs. neutral) ANOVA yielded a significant main effect of epoch $[F(1,14)=24.76, p<.00025]$ and a reliable epoch $\times$ arousal interaction $[F(1,14)=5.56, p<.035]$. To clarify 
A.
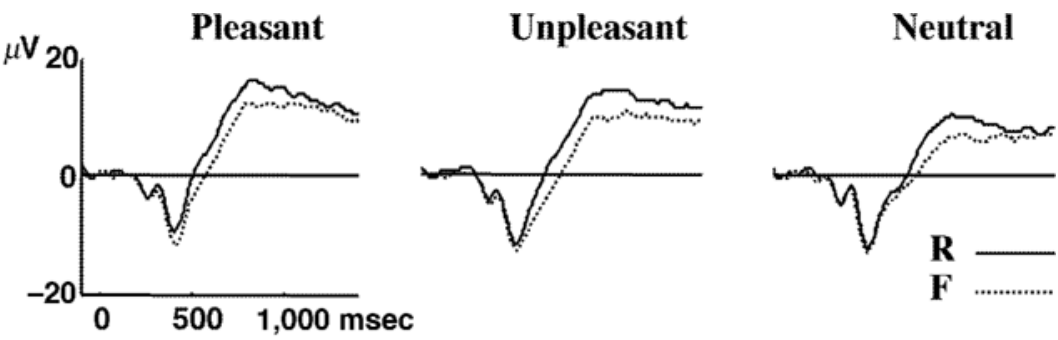

B.
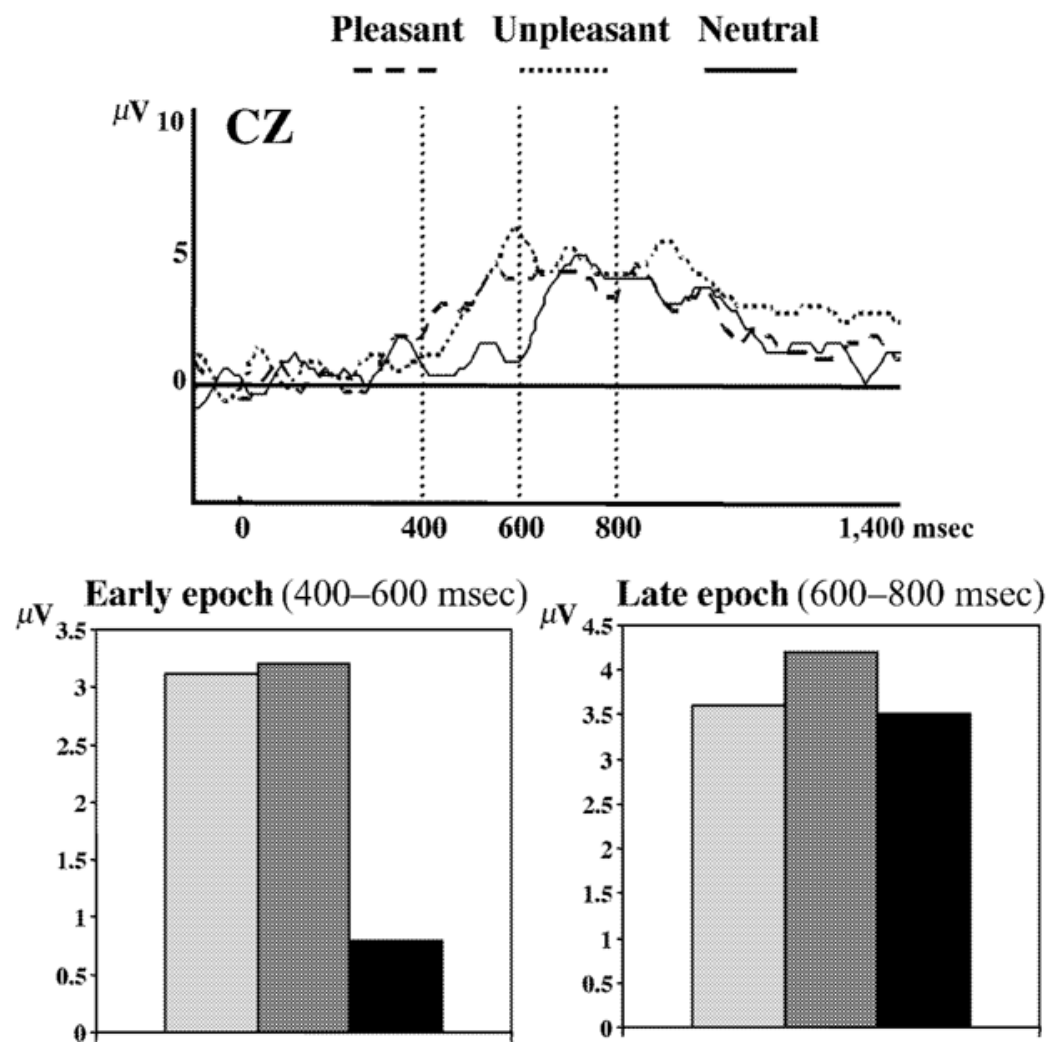

Figure 4. Subsequent memory effect at CZ. (A) Wave graphs comparing the ERPs for remembered (R) and forgotten (F) pictures. (B) Subtraction (recalled - forgotten) wave and bar graphs comparing the subsequent memory effect for pleasant, unpleasant, and neutral pictures. During an early epoch $(400-600 \mathrm{msec})$, the subsequent memory effect was larger for emotional than for neutral pictures. Light gray, pleasant; dark gray, unpleasant; black, neutral.

this interaction, separate ANOVAs were computed on early (400-600 msec) and late (600-800 msec) epochs. A one-way (arousal: emotional vs. neutral) ANOVA showed that the subsequent memory effect was greater for emotional than for neutral pictures in the early epoch $[F(1,14)=$ $6.01, p<.028]$, but not in the late epoch $[F(1,14)=0.1$, $p<.8]$. Consistent with these results, separate ANOVAs directly comparing the waves for remembered and forgotten pictures for each emotion condition (pleasant, unpleasant, and neutral) and epoch (early and late) showed that the subsequent memory effect (remembered vs. forgotten) for both pleasant and unpleasant pictures was significant during both early $[F(1,14)=10.29, p<.0064$, for pleasant, and $F(1,14)=9.56, p<.0081$, for unpleas- ant $]$ and late $[F(1,14)=8.07, p<.0132$, for pleasant, and $F(1,14)=14.14, p<.0022$, for unpleasant] epochs, whereas for neutral pictures, it was significant during the late epoch $[F(1,14)=12.13, p<.0038]$, but not during the early epoch $[F(1,14)=0.85, p<.3732$; see also the remembered/forgotten wave graphs for pleasant, unpleasant, and neutral pictures in Figure 4A]. In other words, the subsequent memory effect at $\mathrm{CZ}$ occurred earlier for emotional than for neutral pictures.

To investigate the possible lateralization of the subsequent memory effect at frontal electrodes, ANOVAs were conducted on ERPs for remembered and forgotten pleasant, unpleasant, and neutral pictures from left $(\mathrm{F} 3)$ and right (F4) electrodes and for both early (400-600 msec) and 
late (600-800 msec) epochs. A 3 (emotion: pleasant, unpleasant, and neutral) $\times 2$ (memory: remembered vs. forgotten) $\times 2$ (hemisphere: left vs. right) repeated measures ANOVA design did not yield any significant hemisphere $\times$ memory, emotion $\times$ memory, or hemisphere $X$ emotion $\times$ memory interactions during the early epoch (400-600 msec); these results suggest that the subsequent memory effect was not different across emotion conditions or hemispheres. However, during the late epoch (600$800 \mathrm{msec}$ ), similar $3 \times 2 \times 2$ ANOVAs yielded significant main effects of both memory $[F(1,14)=27.33, p=.0001]$ and emotion $[F(2,28)=11.01, p=.0003]$ and a significant hemisphere $\times$ emotion $\times$ memory interaction $[F(2,28)=5.68, p<.009]$. Additional ANOVAs meant to elucidate this complex interaction, computed on the subtraction (remembered - forgotten) data, showed that the subsequent memory effect differed across hemispheres only for neutral pictures [left $>$ right; $F(1,14)=5.12, p<$ $.041]$, but not for pleasant $[F(1,14)=.33, p>.575]$ and unpleasant $[F(1,14)=1.55, p>.234]$ pictures. Also, direct comparisons of the subsequent memory effect for pleasant, unpleasant, and neutral pictures did not yield any significant differences, suggesting that, despite the hemispheric differences encountered for neutral pictures, the subsequent memory effect for emotional and neutral pictures was similar at these frontal locations. These results suggest that the ERPs from these locations may reflect comparable involvement of the same frontal neural generators as those associated with successful encoding in functional neuroimaging studies (e.g., Brewer et al., 1998; Wagner et al., 1998).

Collectively, the subsequent memory data show that, as compared with forgotten pictures, remembered pictures elicited larger potentials over centroparietal and frontocentral regions, for both emotional and neutral pictures. However, at central locations (i.e., CZ) and during an early epoch (400-600 msec), the subsequent memory effect was larger for emotional than for neutral pictures, suggesting faster encoding for emotional pictures.

\section{DISCUSSION}

The study yielded two main results, both of which were consistent with our predictions. First, there was a clear topographical difference between arousal-related and valence-related components of the emotion effect: The emotion effect was sensitive to arousal at parietal sites, whereas it was sensitive to both arousal and valence at frontocentral sites. Second, there was a difference between the subsequent memory effects for emotional and neutral pictures: The subsequent memory effect was greater in an early epoch for emotional than for neutral stimuli, suggesting better encoding of high-arousing than of lowarousing stimuli.

\section{Emotion Effect}

At parietal sites, the emotion effect reflected differences in arousal, whereas at frontocentral sites, it reflected differences in both arousal and valence. These findings are consistent with lesion (see Heilman, 2000) and neuroimaging (LaBar et al., 1999) evidence about the role of parietal areas in the processing of emotional arousal and with ERP evidence that the valence-related differences tend to be larger at anterior electrodes than at posterior electrodes (Cuthbert et al., 2000; Diedrich et al., 1997). The findings are also consistent with a neuropsychological model developed by Heller and collaborators (Heller, 1993; Heller \& Nitschke, 1998), which proposes the existence of two distinct neural systems involved in the processing of emotional information: one involved in the processing of emotional arousal located in the parietal lobes, and one involved in the modulation of emotional valence located in the frontal lobes.

It is possible that the ERP differences between emotional and neutral pictures reflected attentional differences that follow from viewing emotional content, rather than direct effects of emotion. The fact that ERPs for emotional and neutral pictures differed mainly in amplitude constitutes evidence for different levels of engagement of the same neural/functional processes (Allan, Wilding, \& Rugg, 1998). Consistent with this idea, there is evidence showing that, because of their motivational significance, emotionally salient stimuli are selected by the brain for sustained attentional processing (see Lang, Simons, \& Balaban, cited in Cuthbert et al., 2000).

The neural generators of ERP differences reported are unclear, but one may speculate that arousal-related differences in the emotion effect primarily reflected the contribution of amygdala-cortical interactions (Amaral et al., 1992), whereas valence-related differences primarily reflected the contribution of the prefrontal cortex (Davidson, 1995; Heller 1993). In addition, because the emotion effect could reflect attentional modulation, these differences may also be related to activity in "attention" areas from parietal and prefrontal regions (e.g., Hopfinger, Buonocore, \& Mangun, 2000).

The suggestion of a possible involvement of the amygdala as the neural generator of the emotion effect at parietal electrodes seems to be in disagreement with evidence suggesting that the amygdala is important in arousalmediated memory effects, rather than in arousal per se (Cahill et al., 1995; LaBar \& Phelps, 1998). That is, it has been shown that amygdala patients did not show enhanced memory to emotional items, despite normal skin conductance responses to emotional words (LaBar \& Phelps, 1998) or normal arousal ratings of emotional scenes of a narrated slide show (Cahill et al., 1995). One possible explanation is that the amygdala is involved in emotional arousal but is not necessary for it. Thus, damage to the amygdala may not impair emotional arousal even if this region participates in emotional arousal in the normal brain (Dolcos, Graham, LaBar, \& Cabeza, in press). Another possible explanation is that the ERP emotion effect from parietal electrodes reflects activity in other brain areas involved in the processing of emotional arousal, such as the parietal lobes. This interpretation is consistent 
with the proposed role of the parietal areas in the processing of emotional arousal (Heller, 1993) and can reconcile the above-mentioned lines of evidence.

The valence-related difference in the emotion effect at frontocentral electrodes could reflect a difference in arousal between the pleasant and the unpleasant pictures, but the fact that pleasant and unpleasant pictures were equated for arousal suggests that this difference could reflect a real valence difference at the cortical level of affective processing (Cuthbert et al., 2000). This difference occurred because the emotion effect started earlier for pleasant than for unpleasant pictures (Figure 2) and could reflect a preference toward pleasant pictures. This interpretation has ecological validity in the sense that, normally, people are more likely to show preference for pleasant than for unpleasant stimuli (the Pollyanna effect; Matlin \& Stang, 1978). It is also consistent with the notion proposed by Diedrich et al. (1997) that the emotion effect in the 400- to 600-msec epoch reflects the processing of the emotional qualities of the stimuli, depending on the amount of attention paid to the emotional content.

This earlier emotion effect for pleasant pictures may seem surprising if one considers the evidence coming from work on the potentiated startle reflex that suggests enhanced response to unpleasant stimuli, relative to pleasant stimuli (e.g., Lang, Bradley, \& Cuthbert, 1990; Schupp, Cuthbert, Bradley, Birbaumer, \& Lang, 1997). A simple explanation of these differences could be that they may reflect different processes. Whereas the affective modulation of the startle reflex is thought of as reflecting motivational priming in which a defensive reflex is augmented when the ongoing motivational state is aversive in nature (Lang, 1995, cited by Schupp et al., 1997), the modulation of the ERP may be interpreted as reflecting earlier processing owing to preference. Given these differences, it would be interesting to design studies intended to dissociate these phenomena.

In sum, the emotion effect results show a clear ERP topographical dissociation of arousal and valence. These results are consistent with previous ERP evidence (Cuthbert et al., 2000; Diedrich et al., 1997) and with evidence supporting the general notion that parietal areas are involved in the processing of emotional arousal (Heilman, 2000; Heller, 1993; LaBar et al., 1999), whereas prefrontal areas are involved in the evaluation of the emotional valence of stimuli (Davidson \& Irwin, 1999; Davidson \& Tomarken, 1989; Heller, 1993).

\section{Subsequent Memory Effect}

The main result of the present study is the finding that the subsequent memory effect was larger, during an early epoch, for emotional than for neutral pictures. There is a considerable amount of evidence that emotional stimuli are processed faster than neutral stimuli (Ohman, Flykt, \& Ludqvist, 2000). If we assume that shorter ERP latencies reflect privileged access to processing resources (Ganis, Kutas, \& Sereno, 1996) and that this advantage in processing leads to better encoding, the present finding of an earlier subsequent memory effect for emotional pictures could be one of the mechanisms underlying better memory for emotional than for neutral events.

However, a question still remains: Why would an earlier engagement of processing resources confer an encoding advantage to emotional stimuli? According to Salthouse (1996), two distinct mechanisms are responsible for the relationship between speed and cognition: the limited time mechanism and the simultaneitymechanism. The limited time mechanism is based on the idea that more processing results in higher performance and that the amount of processing is greater when the speed of processing is faster. The simultaneity mechanism is based on the idea that higher level processing depends on the amount of products of early processing simultaneously available for further processing. Consequently, the faster the speed of processing, the more information that is simultaneously available for high-level processing and, thus, the higher the performance. Adapted to our experiment, these principles could explain our subsequent memory results for high-arousing pictures: The emotional content granted a privileged access to processing resources that resulted in a better encoding of high-arousing pictures. This, in turn, resulted in better retrieval of pleasant and unpleasant pictures, as compared with neutral pictures. If true, this interpretation could explain the beneficial effect of emotion on memory performance.

As for the neural generators of this ERP effect, given the aforementioned pharmacological (Cahill \& McGaugh, 1998; McGaugh et al., 1996), lesion (Adolphs et al., 2000; Cahill et al., 1995; LaBar \& Phelps, 1998), and neuroimaging (Cahill et al., 1996; Canli et al., 2000; Canli et al., 1999; Hamann et al., 1999) evidence linking the amygdala to emotional memory formation, it is reasonable to assume that the earlier subsequent memory effect for emotional pictures indirectly reflects activity in this structure. Although activity in the amygdala is unlikely to volumeconduct to the scalp, this region has intimate connections with a number of neocortical regions (Amaral et al., 1992), and hence, it is likely to influence scalp ERP recordings. The neocortical generators of the emotional modulation of the subsequent memory effect are unclear, but they may involve prefrontal and parietal regions associated with emotional processing (Barbas, 2000; Davidson \& Irwin, 1999; LaBar et al., 1999; Lane et al., 1997; Lang et al., 1998). Finally, the similarity of the subsequent memory effect for emotional and neutral pictures at frontal electrodes may suggest that the ERPs from these locations reflect comparable involvement of the same neural generators as those associated with successful encoding in functional neuroimaging studies (Brewer et al., 1998; Wagner et al., 1998).

\section{Conclusions}

The present study yielded two novel results. First, it yielded a dissociation between arousal and valence. The emotion effect results clearly showed that the processing of the emotional content of pictures is sensitive to both arousal and valence and that their effects can be dissociated over the scalp: ERPs from parietal areas were sen- 
sitive to arousal, whereas ERPs from frontocentral areas were sensitive to both arousal and valence. Second, this is the first study in which the subsequent memory effect for emotional pictorial stimuli has been investigated. The $s u b$ sequent memory effect results show that this effect occurred earlier for pleasant and unpleasant pictures than for neutral pictures and suggest that emotional stimuli have privileged access to processing resources, possibly resulting in better encoding. These results are compatible with previous evidence about the role of different brain areas in the processing of emotional information and with evidence about the beneficial effect of emotion on memory formation. The present study provides the first available evidence of a link between two seemingly unrelated ERP phenomena: the emotion effect and the subsequent memory effect.

\section{REFERENCES}

Adolphs, R., Tranel, D., Damasio, H., \& Damasio, A. (1994). Impaired recognition of emotions in facial expressions following bilateral damage to the human amygdala. Nature, 372, 699-672.

Adolphs, R., Tranel, D., \& Denburg, N. (2000). Impaired emotional declarative memory following unilateral amygdala damage. Learning \& Memory, 7, 180-186.

Allan, K., Wilding, E. L., \& RugG, M. D. (1998). Electrophysiological evidence for dissociable processes contributing to recollection. Acta Psychologica, 98, 231-252.

Amaral, D. G., Price, J. L., Pitkanen, A., \& Carmichael, S. T. (1992). Anatomical organization of the primate amygdaloid complex. In J. P. Aggleton (Ed.), The amygdala: Neurobiological aspects of emotion, memory and mental dysfunction (pp. 1-66). New York: WileyLiss.

BARBAS, H. (2000). Connections underlying the synthesis of cognition, memory, and emotion in primate prefrontal cortices. Brain Research Bulletin, 52, 319-330.

Bechara, A., Damasio, H., Damasio, A. R., \& Lee, P. L. (1999). Different contributions of the human amygdala and ventromedial prefrontal cortex to decision-making. Journal of Neuroscience, 19, 54735481.

Borod, J. C., Koff, E., \& CAron, H. (1983). Right hemisphere specialization for the expression and appreciation of emotion: A focus on the face. In E. Perecman (Ed.), Cognitive processing in the right hemisphere (pp. 83-110). New York: Academic Press.

Borod, J. C., Obler, L. K., Erhan, H. M., Grunwald, I. S., Cicero, B. A., Welkowitz, J., Santschi, C., Agosti, R. M., \& Whalen, J. R. (1998). Right hemisphere emotional perception: Evidence across multiple channels. Neuropsychology, 12, 446-458.

Bradley, M. M., Greenwald, M. K., Petry, M. C., \& Lang, P. J. (1992). Remembering pictures: Pleasure and arousal in memory. Journal of Experimental Psychology: Learning, Memory, \& Cognition, 18, 379-390.

Bradley, M. M., \& LANG, P. J. (1994). Measuring emotion: The selfassessment manikin and the semantic differential. Journal of Behavior Therapy \& Experimental Psychiatry, 25, 49-59.

Brewer, J. B., Zhao, Z., Desmond, J. E., Glover, G. H., \& Gabrieli, J. D. (1998). Making memories: Brain activity that predicts how well visual experience will be remembered. Science, 281, 1185-1187.

CAHILl, L. (1996). Neurobiology of memory for emotional events: Converging evidence from infra-human and human studies. In Cold Spring symposia on quantitative biology (Vol. 61, pp. 259-264). Cold Spring Harbor, NY: Cold Spring Harbor Laboratory Press.

Cahill, L., Babinsky, R., Markowitsch, H., \& McGaugh, J. L. (1995). The amygdala and emotional memory. Nature, 377, 295-296.

Cahill, L., Haier, R. J., Fallon, J., Alkire, M. T., Tang, C., Keator, D., Wu, J., \& McGaugh, J. L. (1996). Amygdala activity at encoding correlated with long-term, free recall of emotional information. Proceedings of the NationalAcademy of Sciences, 93, 8016-8021.
Cahill, L., \& McGaugh, J. L. (1998). Mechanisms of emotional arousal and lasting declarative memory. Trends in Neurosciences, 21, 294-299.

Canli, T., Desmond, J. E., Zhao, Z, Glover, G., \& Gabrieli, J. D. E. (1998). Hemispheric asymmetry for emotional stimuli detected with f MRI. NeuroReport, 9, 3233-3239.

Canli, T., Zhao, Z., Brewer, J., Gabrieli, J. D. E., \& Cahill, L. (2000). Event-related activation in the human amygdala associated with later memory for individual emotional experience. Journal of Neuroscience, 20, 1-5.

Canli, T., Zhao, Z., Desmond, J. E., Glover, G., \& Gabrieli, J. D. E. (1999). fMRI identifies a network of structures correlated with retention of positive and negative emotional memory. Psychobiology, 27, 441-452.

Carretie, L., Iglesias, J., \& Garcia, T. (1997). A study on the emotional processing of visual stimuli through event-related potentials. Brain \& Cognition, 34, 207-217.

Carretie, L., Iglesias, J., Garcia, T., \& Ballesteros, M. (1997). N300, P300 and the emotional processing of visual stimuli. Electroencephalography \& Clinical Neurophysiology, 103, 298-303.

Christianson, S.-A. (1992). The handbook of emotion and memory: Research and theory. Hillsdale, NJ: Erlbaum.

Cuthbert, B. N., Schupp, H. T., Bradley, M. M., Birbaumer, N., \& LANG, P. J. (2000). Brain potentials in affective picture processing: Covariation with autonomic arousal and affective report. Biological Psychology, 52, 95-111.

DAVIDSON, R. J. (1995). Cerebral asymmetry, emotion and affective style. In R. J. H. Davidson \& K. Hugdahl (Eds.), Brain asymmetry (pp. 361387). Cambridge, MA: MIT Press.

DAVIDSON, R. J., \& IRWIN, W. (1999). The functional neuroanatomy of emotion and affective style. Trends in Cognitive Sciences, 3, 11-20.

Davidson, R. J., \& Tomarken, A. J. (1989). Laterality and emotion: An electrophysiological approach. In F. Boller \& J. Grafman (Eds.), Handbook of neuropsychology (Vol. 3, pp. 419-441). New York: Elsevier.

Diedrich, O., Naumann, E., Maier, S., \& Becker, G. (1997). A frontal positive slow wave in the ERP associated with emotional slides. Journal of Psychophysiology, 11, 71-84.

Dolcos, F., Graham, R., LaBar, K. S., \& Cabeza, R. (in press). Coactivation of the amygdala and hippocampus predicts better recall for emotional than for neutral pictures. Brain \& Cognition.

Fabiani, M., Karis, D., \& Donchin, E. (1986). P300 and recall in an incidental memory paradigm. Psychophysiology, 23, 298-308.

Ganis, G., Kutas, M., \& Sereno, M. I. (1996). The search for "common sense": An electrophysiological study of the comprehension of words and pictures in reading. Journal of Cognitive Neuroscience, 8, 89106.

Halgren, E., \& MARinkovic, K. (1995). Neurophysiological networks integrating human emotions. In M. S. Gazzaniga (Ed.), The cognitive neurosciences (pp. 1137-1151). Cambridge, MA: MIT Press.

Hamann, S. B., Ely, T. D., Grafton, S. T., \& Kilts, C. D. (1999). Amygdala activity related to enhanced memory for pleasant and aversive stimuli. Nature Neuroscience, 2, 289-293.

Heilman, K. M. (2000). Emotional experience: A neurological model. In D. R. Lane \& L. Nadel (Eds.), Cognitive neuroscience of emotion (pp. 328-344). New York: Oxford University Press.

HELLER, W. (1993). Neuropsychological mechanisms of individualdifferences in emotion, personality, and arousal. Neuropsychology, 7, 476-489.

Heller, W., \& NitschKe, J. B. (1998). The puzzle of regional brain activity in depression and anxiety: The importance of subtypes and comorbidity. Cognition \& Emotion, 12, 421-447.

Hopfinger, J. B., Buonocore, M. H., \& Mangun, G. R. (2000). The neural mechanisms of top-down attentional control. Nature Neuroscience, 3, 284-291.

Irwin, W., Davidson, R. J., Lowe, M. J., Mock, B. J., Sorenson, J. A., \& TURSKI, P. A. (1996). Human amygdala activation detected with echo-planar functional magnetic resonance imaging. NeuroReport, 7, 1765-1769.

JASPER, H. H. (1958). The ten-twenty electrode system of the International Federation. Electroencephalography \& Clinical Neurophysiology, 10, 371-375. 
Johnston, V. S., Miller, D. R., \& Burleson, M. H. (1986). Multiple P3s to emotional stimuli and their theoretical significance. Psychophysiology, 23, 684-694.

LaBar, K. S., \& Phelps, E. A. (1998). Arousal-mediated memory consolidation: Role of the medial temporal lobe in humans. Psychological Science, 9, 490-493.

LaBar, K. S., Rabinovici, G. D., Ranganath, C., Gitelman, D. R. Parish, T. B., Paller, K. A., \& Mesulam, M.-M. (1999). Spatiotemporal dynamics of a neural network for emotional picture encoding revealed by parallel evoked potential and fMRI measurements. Society for Neuroscience Abstracts, 25, 2146.

LANE, R. D., \& NADEL, L. (2000). Cognitive neuroscience of emotion. New York: Oxford University Press.

Lane, R. D., Reiman, E. M., Bradley, M. M., Lang, P. J., Ahern, G. L., Davidson, R. J., \& Schwartz, G. E. (1997). Neuroanatomical correlates of pleasant and unpleasant emotion. Neuropsychologia, 35, $1437-1444$

LANG, P. J. (1995). The emotion probe: Studies of motivation and attention. American Psychologist, 50, 372-385.

Lang, P. J., Bradley, M. M., \& Cuthbert, B. N. (1990). Emotion, attention, and the startle reflex. Psychological Review, 97, 377-395.

Lang, P. J., Bradley, M. M., \& Cuthbert, B. N. (1997). International affective picture system [Pictures]. Gainesville: NIMH Center for the Study of Emotion and Attention.

Lang, P. J., Bradley, M. M., Fitzsimmons, J. R., Cuthbert, B. N., Scott, J. D., Moulder, B., \& NANGia, V. (1998). Emotional arousal and activation of the visual cortex: An fMRI analysis. Psychophysiology, 35, 199-210.

Lang, P. J., Greenwald, M. K., Bradley, M. M., \& Hamm, A. O. (1993). Looking at pictures: Affective, facial, visceral, and behavioral reactions. Psychophysiology, 30, 261-273.

Lang, P. J., Simons, R. F., \& Balaban, M. T. (1997). Attention and orienting: Sensory and motivational processes. Mahwah, NJ: Erlbaum.

LeDoux, J. E. (1993). Emotional memory systems in the brain. Behavioural Brain Research, 58, 69-79.

Leiphart, J., Rosenfeld, J. P., \& Gabrieli, J. D. (1993). Event-related potential correlates of implicit priming and explicit memory tasks. International Journal of Psychophysiology, 15, 197-206.

Maratos, E. J., Allan, K., \& Rugg, M. D. (2000). Recognition memory for emotionally negative and neutral words: An ERP study. Neuropsychologia, 38, 1452-1465.

Maratos, E. J., \& RUGG, M. D. (2001). Electrophysiological correlates of the retrieval of emotional and non-emotional context. Journal of Cognitive Neuroscience, 13, 877-891.

Matlin, M. W., \& Stang, D. J. (1978). The Pollyanna principle: Selectivity in language, memory, and thought. Cambridge, MA: Shenkman.

McGaugh, J. L., CAHILl, L., \& RoozendaAl, B. (1996). Involvement of the amygdala in memory storage: Interaction with other brain systems. PNAS: Proceedings of the National Academy of Sciences, 93, 13508-13514.

Morris, J. S., Frith, C. D., Perrett, D. I., Rowland, D., Young, A. W., Calder, A. J., \& Dolan, R. J. (1996). A differential neural response in the human amygdala to fearful and happy facial expressions. Nature, 383, 812-815.

Naumann, E., Bartussek, D., Diedrich, O., \& Laufer, M. E. (1992). Assessing cognitive and affective information processing functions of the brain by means of the late positive complex of the event-related potential. Journal of Psychophysiology, 6, 285-298.

Naumann, E., Maier, S., Diedrich, O., Becker, G., \& Bartussek, D. (1997). Structural, semantic, and emotion-focused processing of neutral and negative nouns: Event-related potential correlates. Journal of Psychophysiology, 11, 158-172.

Ohman, A., Fly KT, A. \& LudQvist, D. (2000). Unconscious emotion: Evolutionary perspectives, psychophysiological data and neuropsychological mechanisms. In R. D. Lane \& L. Nadel (Eds.), Cognitive neuroscience of emotion (pp. 296-327). New York: Oxford University Press.

Paller, K. A., Kutas, M., \& Mayes, A. R. (1987). Neural correlates of encoding in an incidental learning paradigm. Electroencephalography \& Clinical Neuropsychology, 67, 360-371.

Palomba, D., ANGrilli, A., \& Mini, A. (1997). Visual evoked potentials, heart rate responses and memory to emotional pictorial stimuli. International Journal of Psychophysiology, 27, 55-67.

Phelps, E. A., \& Anderson, A. K. (1997). Emotional memory: What does the amygdala do? Current Biology, 7, R311-R314.

RugG, M. D. (1995). ERP studies of memory. In M. G. H. Coles \& M. D. Rugg (Eds.), Electrophysiology of mind: Event-related brain potentials and cognition (pp. 132-170). New York: Oxford University Press.

SAlthouse, T. A. (1996). The processing-speed theory of adult age differences in cognition. Psychological Review, 103, 403-428.

Sanguist, T. F., Rohrbaugh, J. W., Syndulko, K., \& Lindsley, D. B. (1980). Electrophysiological signs of level of processing: Perceptual analysis and recognition memory. Psychophysiology, 17, 568-576.

Schneider, F., Grodd, W., Weiss, U., Klose, U., Mayer, K. R., NAGELe, T., \& Gur, R. C. (1997). Functional MRI reveals left amygdala activation during emotion. Psychiatry Research: Neuroimaging Section, 76, 75-82.

Schupp, H. T., Cuthbert, B. N., Bradley, M. M., Birbaumer, N., \& LANG, P. J. (1997). Probe P3 and blinks: Two measures of affective startle modulation. Psychophysiology, 34, 1-6.

SHIELDS, S. (1991). Gender in the psychology of emotion: A selective research review. In K. Strongman (Ed.), International review of studies on emotion (pp. 227-245). New York: Wiley.

VAnderploeg, R. D., Brown, W. S., \& Marsh, J. T. (1987). Judgments of emotion in words and faces: ERP correlates. International Journal of Psychophysiology, 5, 193-205.

Wagner, A. D., Koutstaal, W., \& Schacter, D. L. (1999). When encoding yields remembering: Insights from event-related neuroimaging. Philosophical Transactions of The Royal Society of London: Series $B, 354,1307-1324$.

Wagner, A. D., Schacter, D. L., Rotte, M., Koutstaal, W., Maril, A., Dale, A. M., Rosen, B. R., \& Buckner, R. L. (1998). Building memories: Remembering and forgetting of verbal experiences as predicted by brain activity. Science, 281, 1188-1191.

Wheeler, R. E., Davidson, R. J., \& Tomarken, A. J. (1993). Frontal brain asymmetry and emotional reactivity: A biological substrate of affective style. Psychophysiology, 30, 82-89.

Windmann, S., \& KUTAS, M. (2001). Electrophysiological correlates of emotion-induced recognition bias. Journal of Cognitive Neuroscience, 13, 577-592.

\section{NOTE}

1. The IAPS picture numbers were as follows: pleasant $(2050,4460$, 4470, 4490, 4510, 4520, 4531, 4532, 4533, 4535, 4550, 4561, 4572, $4598,4599,4607,4608,4609,4611,4640,4653,4658,4659,4660$, $4670,4680,4690,5450,5460,5470,5621,5623,5626,5629,5910$, $7270,7400,7502,8021,8030,8031,8034,8041,8080,8090,8130$, $8161,8170,8180,8190,8200,8210,8300,8370,8400,8470,8490$, $8496,8501,8502)$, unpleasant $(1050,1111,1113,1120,1274,1301$, $1310,1930,2053,2730,2800,3051,3062,3061,3150,3180,3220$, $3230,3261,3350,3550,6020,6190,6211,6212,6242,6243,6244$, $6370,6530,6570,6571,6821,6831,7361,7380,8230,8480,9006$, $9040,9042,9050,9160,9181,9250,9300,9420,9490,9561,9570$, $9620,9621,9622,9630,9800,9810,9910,9911,9920,9921)$, and nеutral $(2190,2210,2381,2440,2480,2570,2840,2870,2880,2890$, $4150,4250,4605,5130,5500,5510,5520,5530,5533,5534,5740$, 6150, 7000, 7002, 7004, 7006, 7009, 7010, 7020, 7025, 7030, 7031, $7034,7035,7040,7050,7080,7090,7100,7110,7130,7140,7150$, 7160, 7170, 7175, 7180, 7185, 7187, 7217, 7224, 7233, 7235, 7491, $7500,7550,7710,7950,9210,9700)$.

(Manuscript received December 21, 2001; revision accepted for publication July 19, 2002.) 